\title{
NOTICIAS SOBRE MEDICINA Y SALUD EN UN DIARIO DE DIFUSIÓN NACIONAL. POTENCIAL UTILIDAD EDUCATIVA EN ESTUDIANTES DE CIENCIAS DE LA SALUD
}

\author{
News about medicine and health in a national newspaper. Potential \\ educational use in health sciences students
}

María GONZÁLEZ-GARCÍA; Begoña CANTABRANA; Agustín HIDALGO

Área de Farmacología. Departamento de Medicina. Universidad de Oviedo. Instituto Universitario de Oncología del Principado de Asturias, Fundación CajAstur. Instituto de Investigación Sanitaria de Asturias. Oviedo (España).

e-mail: hidalgo@uniovi.es

Fecha de recepción: 2 de marzo de 2020

Fecha de aceptación: 9 de marzo de 2020

Fecha de publicación: 15 de septiembre de 2020

\begin{abstract}
Resumen
Las noticias de prensa tienen potencial interés formativo en estudiantes de ciencias de la salud por una serie de motivos: a) son frecuentes; b) cubren, al menos parcialmente, la demanda social de información sobre salud; c) aportan información sobre hechos incidentales de forma ágil, lo que favorece el debate crítico; d) permiten la apropiación social del conocimiento propio de las profesiones sanitarias incluyendo la educación para la salud; e) permiten apreciar la difusión del lenguaje médico; f) aportan el contexto sociológico que puede condicionar, al menos en parte, la enfermedad, su imagen social y las medidas que los sistemas públicos ponen en funcionamiento para la atención a los ciudadanos; g) facilitan el desarrollo de competencias de análisis crítico, habilidades de documentación y comunicación; y h) se adaptan bien a la utilización de diferentes metodologías docentes. El artículo propone aspectos de interés formativo a los que pueden contribuir las noticias de prensa, y presenta ejemplos para el abordaje de algunos de ellos.

Palabras clave: noticias de prensa; educación médica; competencias de análisis y comunicación; aspectos sociales de la medicina y los medicamentos.
\end{abstract}


NOTICIAS SOBRE MEDICINA Y SALUD EN UN DIARIO DE DIFUSIÓN NACIONAL.

POTENCIAL UTILIDAD EDUCATIVA EN ESTUDIANTES DE CIENCIAS DE LA SALUD MARÍA GONZÁLEZ-GARCÍA; BEGOÑA CANTABRANA; AGUSTÍN HIDALGO

\begin{abstract}
Press news have a potential educational interest in students of health sciences for several reasons: a) they are frequent; b) cover, at least partially, the social demand for health information; c) provide information on incidental events in an agile manner, which favors critical debate; d) allow the social appropriation of knowledge of health professions including health education; e) allow to know the diffusion of medical language; f) provide the sociological context that can condition, at least in part, the disease, its social image and the measures that the public systems put into operation for the care of citizens; g) facilitate the development of critical analysis skills, documentation and communication skills; and $h$ ) adapt well to the use of different teaching methodologies. The article proposes aspects of formative interest to which press news can contribute, and presents examples for addressing some of them.
\end{abstract}

Key words: press news; medical education; analysis and communication skills; social aspects of medicine and medicines.

\section{Introducción}

La principal función de los medios de comunicación de masas, y en concreto de la prensa escrita, consiste en dar a conocer hechos y opiniones que conforman un marco de convivencia informada. Sus noticias suelen estar redactadas en lenguaje accesible a un amplio número de potenciales lectores, generalmente se refieren a hechos incidentales más que a cuestiones mantenidas en el tiempo exceptuando los reiterados artículos sobre política, utilizan con frecuencia fuentes oficiales y, en los últimos decenios, han otorgado una atención creciente a las noticias sobre ciencia y tecnología o sobre medicina y salud, mayoritariamente con un enfoque de divulgación mediante artículos informativos ${ }^{1}$.

La divulgación científica y sanitaria es indispensable para alcanzar objetivos de apropiación social de la ciencia que contribuirá al incremento de la cultura científica, de apropiación ciudadana de una cultura de salud, de formación de criterio informado y reflexivo, y de formación de una conciencia cívica de acción enfocada tanto al correcto uso de las tecnologías como a la preservación de las conquistas sociales en salud y de afrontamiento de los riesgos de una sociedad tecnificada ${ }^{2-4}$.
De los estudios sobre cultura científica y salud se pueden extraer algunas conclusiones. Entre ellas, que los ciudadanos demandan más información en salud, o que la valoración de científicos y médicos es profesional y socialmente elevada; pero también que utiliza preferentemente fuentes oficiales y que tiene carácter más informativo que educativo. Además, sugiere que la información sobre enfermedades condiciona la percepción que tiene el público sobre la gravedad de las mismas y que una información objetiva y pedagógica contribuye a una mayor comprensión de las mismas por la ciudadanía ${ }^{5-8}$.

Los artículos periodísticos, por otra parte, pueden conformarse como base documental sobre la que realizar lecturas con diferentes objetivos, incluidos los formativos, dada la actualidad de los hechos contenidos en los mismos ${ }^{9,10}$. De la misma forma, también se han planteado actividades formativas con materiales no habituales en la enseñanza de la medicina; entre ellos, el cine y las series de televisión ${ }^{11,12}$, el arte ${ }^{13}$ o el cómic ${ }^{14}$.

En una editorial de esta misma revista hemos hecho una aproximación a la potencial utilidad formativa de las noticias de prensa ${ }^{15}$. Describimos a continuación las características de la base documental que hemos confeccionado y aportamos algunos ejemplos de los diferentes apartados en

Rev. Med. Cine. 2020; 16(3), 223-234 Ediciones Universidad de Salamanca / @@ J. Med. Mov., 2020; 16 (3), $223-234$ 
NOTICIAS SOBRE MEDICINA Y SALUD EN UN DIARIO DE DIFUSIÓN NACIONAL.

POTENCIAL UTILIDAD EDUCATIVA EN ESTUDIANTES DE CIENCIAS DE LA SALUD MARÍA GONZÁLEZ-GARCÍA; BEGOÑA CANTABRANA; AGUSTÍN HIDALGO

los que consideramos que su contribución puede ser relevante.

\section{Material y Métodos}

Elaboración de la colección. Las noticias utilizadas en el artículo han sido extraídas de la colección CONPRE Oviedo (Colección de noticias de prensa. Universidad de Oviedo), una base documental de noticias relacionadas con la ciencia y la tecnología, la medicina y la salud, los medicamentos, la sociología y la educación, así como la terminología médica, cartas al editor y viñetas de humor, que contiene 7688 artículos periodísticos. Una parte significativa de los mismos se relacionan con aspectos que concurren en la práctica de la medicina, tales como sus componentes científicos, sus estrategias terapéuticas, la formación de profesionales sanitarios, la dimensión global de la salud y de la enfermedad, su trascendencia y repercusión social (visibles en el humor, en la incorporación del lenguaje médico al lenguaje cotidiano, etc.) así como los componentes sociopolíticos y económicos implicados en la organización de los sistemas sanitarios.

Como fuente documental principal para la elaboración de la colección se utilizó la edición nacional impresa del diario El País, siendo el periodo de recogida de información desde el 2 de enero de 2001 a 31 de diciembre de 2016. La recogida se ha llevado a cabo por un único observador lo que se reconoce como un factor limitante del estudio. La colección contiene noticias ocasionales del diario La Nueva España (el periódico local de Asturias con mayor tirada regional y octavo en difusión a nivel nacional) y, esporádicamente, de otros diarios de ámbito nacional o regional.

Criterios de selección. Los criterios de selección de las noticias, han sido los siguientes ${ }^{16}$ : a) el título debe aludir a términos de interés para la colección (fundamentalmente ciencia y tecnología, medicina y salud, medicamentos, educación universitaria, sociología, terminología médica, humor y comunicación de los pacientes mediante cartas al editor), b) tener un autor identificable, c) estar basada en hechos reales, d) tener entidad narrativa y e) contener una historia completa. Es decir, debe identificar y delimitar un tema, explicitar los factores concurrentes $y$, si es posible, aproximar una posible evolución del mismo. No se han considerado las noticias procedentes de agencias sin un autor identificable, ni se han incluido las aparecidas en suplementos semanales o en fascículos monográficos a menos que estuvieran relacionadas con un tema de repercusión emergente en el diario en fechas próximas.

Distribución en apartados. Las noticias se agruparon por anualidades en el periodo 2001-2016 y se distribuyeron en apartados y sub-apartados por, al menos, dos observadores independientes, consensuando la decisión en casos de dudas. Algunas noticias han sido asignadas a más de un área de interés o a diferentes sub-apartados dentro de la misma área. En todo caso, en los datos globales que se presentan en este trabajo, cada noticia está considerada una única vez. Por otra parte, la propuesta de los aspectos formativos en los que pueden ser útiles las noticias de prensa es fruto de la consideración de los aspectos tratados por la prensa y de la discusiones y síntesis por los autores del artículo.

Potencial formativo. Con el fin de explorar el potencial formativo en estudiantes de medicina en la tabla 1 proponemos algunas áreas en las que las noticias de prensa pueden ser útiles y, en el capítulo de resultados, desarrollamos algunos ejemplos orientativos de cómo transformar una noticia de prensa en una actividad formativa.

Análisis estadístico. Se ha realizado una distribución de frecuencias mostrando los datos como valores absolutos.

\section{Resultados}

\section{Distribución y evolución de las noticias}

Al realizar la distribución de artículos que contiene la colección CONPRE Oviedo, se observa que el $76,6 \%(n=5.894)$ corresponden a tres apartados a) Medicina y Salud ( $n=2.001$; artículos

Rev. Med. Cine. 2020; 16(3), 223-234 Ediciones Universidad de Salamanca / @@@ J. Med. Mov., 2020; 16 (3), $223-234$ 
NOTICIAS SOBRE MEDICINA Y SALUD EN UN DIARIO DE DIFUSIÓN NACIONAL.

POTENCIAL UTILIDAD EDUCATIVA EN ESTUDIANTES DE CIENCIAS DE LA SALUD MARÍA GONZÁLEZ-GARCÍA; BEGOÑA CANTABRANA; AGUSTÍN HIDALGO

que ofrecen información sobre enfermedad, su evolución o regulación y/o nuevo conocimiento fundamental, diagnóstico o terapéutico, aplicado a la medicina), b) Medicamentos ( $n=1.960$; artículos sobre cualquier aspecto relacionado con una molécula destinada a uso clínico que aplique a características farmacológicas, reguladoras o sociales de los mismos) y c) Ciencia y Tecnología ( $\mathrm{n}=1.933$; artículos cuyo propósito es comunicar nuevo conocimiento científico y/o tecnológico que implica avance en las ciencias o potencial aplicación a la salud). Por otra parte, las noticias sobre Educación (artículos que comunican aspectos relacionados con la educación universitaria, formación sanitaria especializada, reformas y calidad educativa) ocupan el cuarto lugar, $(n=810)$ seguidas de las referidas a Sociología ( $n=318$; artículos referidos a aspectos de la medicina y de la ciencia que tienen una dimensión global (política, económica, social) y que no se clasifica en otros apartados) y
Terminología médica ( $\mathrm{n}=318$; artículos generales de información no especializada en los que se incluyan términos propios de la medicina para aludir a cuestiones no médicas), Humor ( $n=226$; viñetas de los humoristas del diario a lo largo del periodo de estudio que aludan a aspectos de la medicina, la ciencia, los medicamentos, la gestión clínica, etc.) y Cartas al Editor ( $n=122)$ texto de un lector dirigido al diario para manifestar su opinión sobre diferentes aspectos de la medicina).

Como muestra la figura 1, las noticias de cada uno de los grupos tienen variabilidad anual lo que conforma el aspecto de evolución en «diente de sierra" con picos que responden a la acumulación de noticias incidentales y valles en los que se acumulan las noticias crónicas o mantenidas en el tiempo. La figura también muestra como el número de noticias seleccionadas es creciente a lo largo del periodo de observación, sobre todo en los últimos años.

Figura 1. Evolución temporal del número de noticias de los diferentes apartados de la colección CONPRE Oviedo.

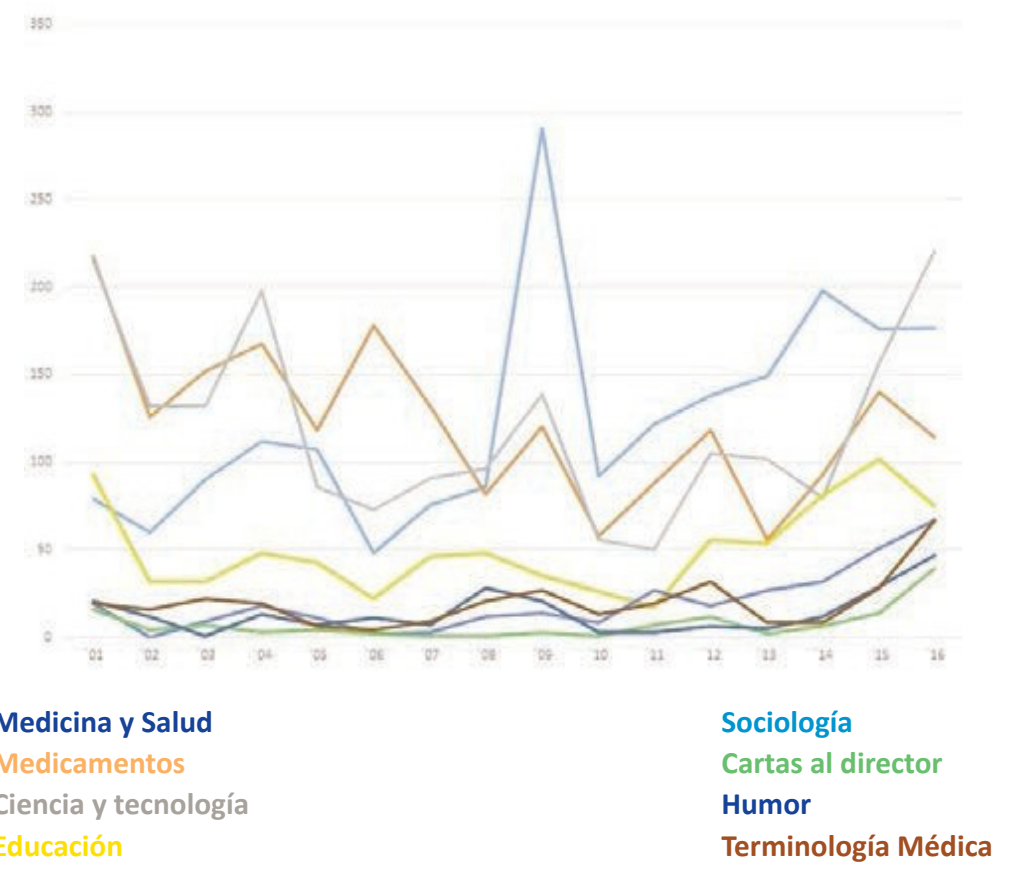

Rev. Med. Cine. 2020; 16(3), 223-234 Ediciones Universidad de Salamanca / @®@ 
NOTICIAS SOBRE MEDICINA Y SALUD EN UN DIARIO DE DIFUSIÓN NACIONAL.

POTENCIAL UTILIDAD EDUCATIVA EN ESTUDIANTES DE CIENCIAS DE LA SALUD MARÍA GONZÁLEZ-GARCÍA; BEGOÑA CANTABRANA; AGUSTÍN HIDALGO

Tabla 1. Ejemplos de potencial utilidad docente de las noticias de prensa.

\begin{tabular}{|c|c|c|}
\hline Aspecto formativo & Utilidad docente & Ejemplo de noticia \\
\hline $\begin{array}{l}\text { 1. Conocer los avances } \\
\text { de la ciencia en general } \\
\text { y su aplicación a la me- } \\
\text { dicina }\end{array}$ & $\begin{array}{l}\text { Aspectos científicos y sanitarios emergentes. } \\
\text { La contribución a la historia de la ciencia. } \\
\text { La traslación de la investigación a la clínica. } \\
\text { La contribución a formar una cultura cientí- } \\
\text { fica y sanitaria. }\end{array}$ & $\begin{array}{l}\text { - Emilio de Benito. Un nuevo éxito reaviva } \\
\text { las esperanzas en la terapia génica. } 16 \text { sep- } \\
\text { tiembre } 2010 \text {. } \\
\text { - Emilio de Benito. Descubierto el gen que } \\
\text { vuelve agresivo el cáncer de próstata. } 10 \text { fe- } \\
\text { brero } 2015 \text {. }\end{array}$ \\
\hline $\begin{array}{l}\text { 2. Adquisición de len- } \\
\text { guaje médico }\end{array}$ & $\begin{array}{l}\text { Introducción al lenguaje médico. } \\
\text { Adquisición de vocabulario. } \\
\text { Conocer la penetración social del lenguaje } \\
\text { médico. } \\
\text { Conocer el uso metafórico de términos mé- } \\
\text { dicos para designar realidades sociales. }\end{array}$ & $\begin{array}{l}\text { - Charo Nogueira. Pandemia de pesimismo. } \\
12 \text { mayo } 2012 . \\
\text { - Vicente Verdú. Metástasis de la vida coti- } \\
\text { diana. } 19 \text { enero } 2008 .\end{array}$ \\
\hline $\begin{array}{l}\text { 3. Como fuente docu- } \\
\text { mental para seminarios }\end{array}$ & $\begin{array}{l}\text { Facilita una estrategia de educación en re- } \\
\text { pertorios de documentación científica. } \\
\text { Los estudiantes pueden realizar una selec- } \\
\text { ción de textos, una contextualización y la } \\
\text { extracción de conclusiones. }\end{array}$ & $\begin{array}{l}\text { - Gonzalo Casino. Osteoporosis, ¿Una epi- } \\
\text { demia inventada? } 25 \text { de abril de } 2006 \text {. } \\
\text { - Jaime Prats. Sanidad suspende la comer- } \\
\text { cialización de } 29 \text { medicamentos genéricos. } \\
27 \text { enero } 2015 .\end{array}$ \\
\hline $\begin{array}{l}\text { 4. Organizar foros de } \\
\text { discusión sobre temas } \\
\text { de diferentes asignatu- } \\
\text { ras }\end{array}$ & $\begin{array}{l}\text { Repercusiones ecológicas, efectos lobistas, } \\
\text { efectos económicos o papel de la publicidad } \\
\text { en el consumo de medicamentos. } \\
\text { Repercusiones de la tecnología en la salud. } \\
\text { Medidas legislativas en la salud, etc. }\end{array}$ & $\begin{array}{l}\text { - David Segarra. Medicamentos en el río. } 17 \\
\text { enero } 2006 . \\
\text { - Rafael Méndez, Carmen Morán. Indemni- } \\
\text { zación para las víctimas de la talidomida } 40 \\
\text { años después. } 23 \text { marzo } 2010 .\end{array}$ \\
\hline $\begin{array}{l}\text { 5. Resolver problemas } \\
\text { científicomédicos plan- } \\
\text { teados en las noticias }\end{array}$ & $\begin{array}{l}\text { Fraude y Toxicidad de medicamentos. Incer- } \\
\text { tidumbres de procedimientos terapéuticos. } \\
\text { Causas de hechos sociales de gran repercu- } \\
\text { sión. Variabilidad biológica. } \\
\text { Interacciones medicamentosas. }\end{array}$ & $\begin{array}{l}\text { - Lluis Visa. } 19 \text { detenidos por vender o usar } \\
\text { sustancias de engorde ilegal de ganado. } 20 \\
\text { enero } 2004 . \\
\text { - María R. Sahuquillo. La píldora del día } \\
\text { siguiente pierde eficacia en obesas. } 27 \text { no- } \\
\text { viembre } 2013 \text {. }\end{array}$ \\
\hline $\begin{array}{l}\text { 6. Analizar la influencia } \\
\text { del Contexto sociológi- } \\
\text { co en la salud }\end{array}$ & $\begin{array}{l}\text { Factores determinantes de la política educa- } \\
\text { tiva y de la política sanitaria. } \\
\text { Factores culturales implicados en la salud. } \\
\text { Nuevos consensos morales provocados por } \\
\text { el avance de las ciencias y sus repercusiones } \\
\text { en salud. }\end{array}$ & $\begin{array}{l}\text { - Emilio de Benito. Los parados se drogan } \\
\text { más. } 27 \text { enero de } 2011 . \\
\text { - E. G. Sevillano \& M. Marín. Sanidad pagará } \\
\text { hasta } 25.000 \text { euros por la «penicilina» de la } \\
\text { hepatitis C. } 25 \text { septiembre } 2014 \text {. }\end{array}$ \\
\hline $\begin{array}{l}\text { 7. Abordar competen- } \\
\text { cias transversales o } \\
\text { huérfanas en la forma- } \\
\text { ción del médico. }\end{array}$ & $\begin{array}{l}\text { Es importante porque: } \\
\text { No suelen definirse en los planes de estu- } \\
\text { dios, ni evaluarse en la práctica. } \\
\text { Incluyen aspectos poco presentes en los pla- } \\
\text { nes de estudios. } \\
\text { Se relacionan con los valores de la profesión }\end{array}$ & $\begin{array}{l}\text { - Javier Sampedro. No se olviden del enfer- } \\
\text { mo. } 25 \text { de julio de } 2006 \text {. } \\
\text { - Yolanda Monge. EEUU pide perdón a Gua- } \\
\text { temala por experimentar con pacientes y } \\
\text { presos. } 2 \text { octubre } 2010 .\end{array}$ \\
\hline $\begin{array}{l}\text { 8. Conocer la imagen } \\
\text { social del profesional } \\
\text { sanitario a través de } \\
\text { opiniones de usuarios, } \\
\text { asociaciones de } \\
\text { pacientes, etc. }\end{array}$ & $\begin{array}{l}\text { Planean reivindicaciones sociales. } \\
\text { Demandan logros sociales en salud. } \\
\text { Pueden condicionar políticas sanitarias. } \\
\text { Configuran una imagen social del médico. } \\
\text { Conforman una imagen de la actividad } \\
\text { asistencial del sistema de salud. } \\
\text { Informa de la repercusión social de las } \\
\text { actuaciones profesionales }\end{array}$ & $\begin{array}{l}\text { - Carmelo Vázquez. Vidas medicables. } 25 \\
\text { marzo } 2007 . \\
\text { - Luis Palomo. El gasto sanitario. } 8 \text { agosto } \\
2011 . \\
\text { - Emilio de Benito, María Lillo. Los enfermos } \\
\text { «raros» toman las riendas. } 27 \text { febrero } 2014 . \\
\text { - Viñeta de El Roto. Un personaje: ¿Qué } \\
\text { te ha dicho el psiquiatra? El otro: Que si le } \\
\text { podía prestar } 20 € .17 \text { julio } 2013 \text {. }\end{array}$ \\
\hline
\end{tabular}

Rev. Med. Cine. 2020; 16(3), 223-234 Ediciones Universidad de Salamanca / @@@ J. Med. Mov., 2020; 16 (3), $223-234$ 
NOTICIAS SOBRE MEDICINA Y SALUD EN UN DIARIO DE DIFUSIÓN NACIONAL.

POTENCIAL UTILIDAD EDUCATIVA EN ESTUDIANTES DE CIENCIAS DE LA SALUD MARÍA GONZÁLEZ-GARCÍA; BEGOÑA CANTABRANA; AGUSTÍN HIDALGO

Por otra parte, si bien los aspectos identificados cada año mantienen un nivel importante de noticias (más de 100 noticias de media cada año a lo largo del periodo de estudio), es evidente que las relacionadas con Ciencia y Tecnología, Medicina y Salud, y Medicamentos presentan notables oscilaciones de unos años a otros relacionadas, generalmente, con hechos incidentales. De hecho, el gran pico del año 2009 corresponde a la Gripe A (H1N1), y el del año 2014 a la epidemia de Ébola. Así mismo, hay dos picos que corresponden a noticias sobre el aborto en los años 2009 y 2013. En el caso de los medicamentos, las variaciones se producen de forma importante de un año a otro.

En el resto de los apartados el número de noticias es más homogéneo, tal vez con la excepción de las referidas a las modificaciones de los planes de estudios universitarios, emergencia de universidades privadas y modificaciones educativas introducidas a partir de 2012 por el Gobierno presidido por Mariano Rajoy.

\section{Potencial educativo de las noticias en ciencias de la salud}

Más allá de los datos cuantitativos de las noticias de la colección, dentro de los diferentes apartados pueden encontrarse noticias útiles para plantear estrategias formativas en aspectos de la salud poco o nada presentes en los curricula académicos de los Grados en Ciencias de la Salud.

En la tabla 1 recogemos algunas propuestas formativas y aspectos docentes que pueden ser elaborados bajo cada uno de los epígrafes. Así mismo y de forma orientativa, ofrece algunos ejemplos de noticias relacionadas con cada uno de ellos.

Naturalmente, la relación de aspectos docentes que pueden abordarse dentro de cada apartado no pretende ser exhaustivo sino únicamente orientativo y, más allá de los incluidos en la tabla 1, mayoritariamente de Medicina y Salud, y de Medicamentos, puede recurrirse a noticias de otros grupos temáticos. Por otra parte, para la resolución de algunas cuestiones es necesario que el estudiante tenga nociones elementales de farmacología mientras que para otros no se requiere una instrucción específica más allá de los conocimientos propios de las asignaturas de los primeros cursos de los grados en Ciencias de la Salud.

Naturalmente, el análisis del hecho noticiable no debe circunscribirse al momento en que se produce la noticia sino que debe procurar seguir la evolución temporal de la misma y, cuando sea posible, aportar una conclusión del hecho y una explicación razonada a la resolución del problema suscitado.

\section{Algunos ejemplos de la relación entre noticias de prensa y actividad formativa}

1. Un caso de interacción medicamentosa con potencial agravamiento de una patología previa con resultado de muerte.

Un artículo de Isabel Ferrer ${ }^{17}$ en el diario El País del día 14 de marzo de 2006 informa de la muerte del líder serbio Slobodan Milosevic en una prisión del Tribunal Penal Internacional. Aparentemente el político observaba un cumplimiento terapéutico errático posiblemente para forzar su traslado a Rusia donde residía su familia y él podría quedar en libertad. Al morir de un infarto y detectarse la presencia de un medicamento que no se le había prescrito, el antibiótico rifampicina, se especula con la posibilidad de que Milosevic pueda haber sido asesinado o se haya suicidado mediante el uso de tal medicamento que no le ha sido prescrito por los médicos responsables de su salud en la prisión.

La tarea que se puede plantear a propósito de esta noticia permite animar a los estudiantes (Tabla 2) a relatar la relación entre hipertensión e infarto de miocardio, identificar los medicamentos que podría estar tomando, el perfil farmacológico de la rifampicina, las razones de la potencial 
NOTICIAS SOBRE MEDICINA Y SALUD EN UN DIARIO DE DIFUSIÓN NACIONAL.

POTENCIAL UTILIDAD EDUCATIVA EN ESTUDIANTES DE CIENCIAS DE LA SALUD MARÍA GONZÁLEZ-GARCÍA; BEGOÑA CANTABRANA; AGUSTÍN HIDALGO

responsabilidad del antibiótico en la muerte y el mecanismo por el que pueden contribuir a la misma. La resolución esperable es que el efecto inductor enzimático de la rifampicina aumente el metabolismo hepático de los antihipertensivos, reduzca su eficacia y favorezca el fracaso cardíaco y la aparición del infarto de miocardio, como explica el doctor Pedro Mata ${ }^{18}$ en el mismo diario.

Tabla 2. Un caso de potencial interacción medicamentosa con resultado de muerte.

\begin{tabular}{|c|c|}
\hline Noticia & Tareas del estudiante \\
\hline $\begin{array}{l}\text { El líder serbio Slobodan Milosevic fue } \\
\text { encontrado muerto en su celda de una } \\
\text { cárcel del Tribunal Penal Internacional en } \\
\text { Holanda. La autopsia reveló que la causa } \\
\text { de la muerte fue un infarto de miocardio, } \\
\text { que ocurrió después de que el tribunal } \\
\text { rechazara su traslado a Rusia para una in- } \\
\text { tervención cardiaca, y que en la sangre del } \\
\text { cadáver se habían encontrado trazas de } \\
\text { Rifampicina, un medicamento que nunca } \\
\text { le fue prescrito oficialmente por los médi- } \\
\text { cos que trataban su hipertensión. }\end{array}$ & $\begin{array}{l}\text { - Establecer un perfil biográfico y político de Milosevic. } \\
\text { - Identificar su enfermedad de base, su posible tratamiento y su } \\
\text { cumplimiento terapéutico. } \\
\text { - Establecer la relación entre hipertensión e infarto de miocardio y } \\
\text { describir la fisiopatología del proceso. } \\
\text { - Identificar el grupo terapéutico de la rifampicina, sus indicaciones } \\
\text { autorizadas y características cinéticas. } \\
\text { - Explicar el comportamiento de la rifampicina sobre el metabolismo } \\
\text { hepático de sustancia químicas. } \\
\text { - Explicar la potencial responsabilidad de la rifampicina en la muerte de } \\
\text { Milosevic. }\end{array}$ \\
\hline
\end{tabular}

Tabla 3. Una propuesta de tarea sobre crisis económica y enfermedad social.

\begin{tabular}{|c|c|}
\hline Resumen del artículo (Noriega, 2012) & Tareas para los estudiantes \\
\hline $\begin{array}{l}\text { La situación de crisis económica que se } \\
\text { inició en el año } 2008 \text { y se ha extendido } \\
\text { durante más de un decenio ha provo- } \\
\text { cado cambios sociales con aumento del } \\
\text { desempleo, inestabilidad social, cambios } \\
\text { en los estilos de vida y en las emocio- } \\
\text { nes de los ciudadanos hasta el punto de } \\
\text { que se ha convertido en una verdadera } \\
\text { enfermedad social. En este contexto, par- } \\
\text { te de la ciudadanía ha visto incrementa- } \\
\text { da su vulnerabilidad emocional y se ha } \\
\text { acentuado el trinomio ansiedad, ira y } \\
\text { depresión que han motivado aumento } \\
\text { de la frecuentación de servicios y mayor } \\
\text { consumo de recursos sanitarios. }\end{array}$ & $\begin{array}{l}\text { - Documentar los términos crisis, pandemia, ansiedad, ira y depresión; } \\
\text { establecer sus significados en el contexto del artículo y en el campo de } \\
\text { la medicina. } \\
\text { - Explicar los conceptos de enfermedad social y retraimiento social e } \\
\text { identificar las condiciones que los convierten en patológicos. } \\
\text { - Explicar las razones por las que el desempleo y la pobreza incrementan } \\
\text { la morbilidad y mortalidad. } \\
\text { - Explicar si, en su opinión, la crisis económica ha contribuido a la } \\
\text { aparición de aporofobia. }\end{array}$ \\
\hline
\end{tabular}

\section{Relación entre crisis económica y salud}

Charo Noriega ${ }^{19}$ elaboró un artículo basado en consultas a especialistas médicos, psicólogos y sociólogos, en el que retrata la «pandemia de pesimismo" de la sociedad española en el año 2012, en plena crisis económica. Sostienen los profesionales consultados que la situación de crisis ha provocado pesimismo social asentado en la falta de expectativas y en la convicción de que no hay salidas a la crisis y que, además, desde el exterior nos inducen a asumir un sentimiento de culpa porque somos responsables y debemos pagar por ello. También resalta, como indica la tabla 3, que el riesgo de ansiedad y depresión aumenta entre los parados y que la situación de desempleo mantenida genera el trinomio ansiedad, ira y depresión que lleva aparejada una mayor frecuentación de los servicios sanitarios.

Rev. Med. Cine. 2020; 16(3), 223-234 Ediciones Universidad de Salamanca / @®@ J. Med. Mov., 2020; 16 (3), $223-234$ 
NOTICIAS SOBRE MEDICINA Y SALUD EN UN DIARIO DE DIFUSIÓN NACIONAL.

POTENCIAL UTILIDAD EDUCATIVA EN ESTUDIANTES DE CIENCIAS DE LA SALUD MARÍA GONZÁLEZ-GARCÍA; BEGOÑA CANTABRANA; AGUSTÍN HIDALGO

3. Medicamentos huérfanos para enfermos «Raros»

Emilio de Benito y María Lillo ${ }^{20}$ (2014) firman un artículo sobre enfermedades raras en el que, con motivo de la celebración del Año de las Enfermedades Raras, dedican un análisis divulgativo del estado de la cuestión con participación de científicos y asociaciones de pacientes. Resaltan que los nuevos avances en genética y en biotecnología hacen posible plantearse intervenciones potencialmente curativas en algunas de las 7000 enfermedades raras estimadas mediante la administración de una versión correcta del gen defectuoso. El artículo transmite optimismo basado en la existencia de centros de investigación que pueden abordar esta compleja tecnología, científicos bien formados, capaces y con entusiasmo, acciones de las Agencias Reguladoras de medicamentos que facilitan el desarrollo de medicamentos para enfermedades raras, la existencia de un CIBER singular dedicado a estas patologías, la organización de asociaciones de pacientes, o la creciente importancia del micromecenazgo en la aportación de recursos financieros orientados a la investigación en este campo. Esta noticia permite abordar una serie de cuestiones de frontera en el campo de la investigación sobre enfermedades poco prevalentes como muestra la tabla 4.

\section{El enfermo en el centro de la atención sanitaria}

Javier Sampedro ${ }^{21}$, uno de los grandes divulgadores científicos de nuestro país, titula un artículo, que se remonta al año 2006, «no se olviden del enfermo", en el que glosa diversas iniciativas formativas del Centro Nacional de Investigación Cardiovascular (CNIC) tanto con estudiantes de enseñanzas secundarias como con residentes de cardiología. En este artículo se hace eco de algunas reflexiones que atribuye al Dr. Valentín Fuster, director científico del CNIC, y que consideramos de interés para la formación de los estudiantes de ciencias de la salud.

Tabla 4. Propuesta de ejercicio sobre la emergencia científica y social de las enfermedades raras.

\begin{tabular}{|c|c|}
\hline Resumen del artículo (De Benito y Lillo, 2014) ${ }^{20}$ & Tareas para los estudiantes \\
\hline $\begin{array}{l}\text { El desarrollo de la genética molecular ha } \\
\text { facilitado plantearse el abordaje terapéutico de } \\
\text { enfermedades de baja prevalencia, las denominados } \\
\text { enfermedades raras y ultra-raras, como punta } \\
\text { de lanza de tratamientos personalizados de base } \\
\text { genética, ya que el } 80 \% \text { de estas enfermedades } \\
\text { son genéticas. El hecho de que un porcentaje } \\
\text { sea debido a alteraciones monogénicas y que se } \\
\text { hayan conseguido algunos éxitos en enfermedades } \\
\text { metabólicas debidas a carencia de un gen, hace } \\
\text { concebir esperanzas de que, en el futuro, puedan } \\
\text { curarse un número significativas de ellas, aunque } \\
\text { el camino está lleno de dificultades y es necesaria } \\
\text { tanto la colaboración institucional como ciudadana. }\end{array}$ & $\begin{array}{l}\text { - Conceptualizar «Enfermedad Rara» y cuantificar el problema } \\
\text { en España. } \\
\text { - Explicar sus bases genéticas. } \\
\text { - Explicar los procesos que van de la alteración genética a la } \\
\text { enfermedad. } \\
\text { - Describir la actitud de las autoridades españolas y europeas } \\
\text { ante el problema. } \\
\text { - Describir el papel potencial de la terapia génica en el } \\
\text { tratamiento convencional de la enfermedad. } \\
\text { - Explicar el papel que atribuyen a los biobancos en la } \\
\text { investigación. } \\
\text { - Definir el papel que asumen las asociaciones de pacientes y } \\
\text { el micromecenazgo. } \\
\text { - Sugerir otras actuaciones de interés. }\end{array}$ \\
\hline
\end{tabular}

Es obvio que la medicina ha ganado en eficacia gracias al desarrollo de las ciencias, a medida que se ha hecho científica y ha incorporado productos tecno-científicos a la práctica clínica. Esto ha tenido una especie de efecto llamada para los profesionales sanitarios que se han convertido a la técnica olvidando que "la tecnología se puede aprender en cualquier momento» y que lo más crítico de 
NOTICIAS SOBRE MEDICINA Y SALUD EN UN DIARIO DE DIFUSIÓN NACIONAL.

POTENCIAL UTILIDAD EDUCATIVA EN ESTUDIANTES DE CIENCIAS DE LA SALUD MARÍA GONZÁLEZ-GARCÍA; BEGOÑA CANTABRANA; AGUSTÍN HIDALGO

la medicina real es «entender el problema del paciente», es decir, conocer al enfermo en todas sus dimensiones, incluyendo, naturalmente, los aspectos humanos.

Esta apreciación es particularmente importante en la actualidad dado que la actividad médica ha experimentado una tecnificación creciente y las orientaciones formativas tanto en el grado como durante la residencia MIR contemplan la formación en investigación ${ }^{22}$. El artículo, en consecuencia, puede servir tanto para plantear las características que debe reunir un investigador ( «libertad, altruismo, pasión y consistencia»), a los que puede añadirse la constancia, la relación entre ciencia y humanismo en la práctica de la medicina, su repercusión en el abordaje de la relación médicopaciente y, sobre todo, en la necesidad de que se mantenga al enfermo en el centro de la atención sanitaria.

\section{Discusión}

La base de datos que hemos confeccionado dispone de un número apreciable de noticias, de las que las relacionadas con ciencia y tecnología, medicina y salud y medicamentos suponen la mayor parte. En conjunto suponen más de 100 noticias al año lo que supone que, al menos estos tres apartados, tienen un peso importante en la noticiabilidad cotidiana, por lo que podemos considerar que la edición impresa de El País contribuye tanto a la demandada comunicación social de la ciencia ${ }^{2}$, como a la información en salud, incluyendo los medicamentos, algo que ya se ha avanzado por otros autores ${ }^{1,7} y$ hemos comunicado en un artículo previo ${ }^{16}$.

Si bien la mayoría de las noticias proceden de un único diario y han sido seleccionadas originariamente por un único autor, lo que puede considerarse como una debilidad del trabajo, no es menos cierto que se trata del diario de mayor difusión de nuestro país y que la recogida longitudinal a lo largo de 16 años, nos permitirá hacer un seguimiento de la evolución de los temas y del tratamiento de los mismos a lo largo del periodo. La colección, como ya hemos comentado en otra comunicación ${ }^{16}$, aun siendo más limitada que otras similares ${ }^{1}$, permite observar algunas características tales como la evolución en dientes de sierra o la existencia de un perfil agudo (o incidental) y otro crónico (o mantenido) de las noticias, con preeminencia de las noticias de alto impacto periodístico y/o social sobre las médica o científicamente relevantes, lo que confiere carácter social a la prensa y supone la base de la orientación formativa que pueden aportar al perfil de egreso de los estudiantes de ciencias de la salud.

Otro aspecto noticiable de nuestra colección es que el número de noticias seleccionadas de ciencia y tecnología, de medicina y salud y de medicamentos es muy similar lo que sugiere que la comunicación en estas tres áreas se ha incorporado de forma decidida a la tarea de los medios de redacción.

En la tabla 1 hemos aportado propuestas de 8 actividades en las que las noticias de prensa pueden contribuir a la formación de estudiantes de titulaciones de Ciencias de la Salud, a las que hemos asignado algunas referencias potencialmente útiles. También a modo de ejemplo hemos desarrollado algunos ejemplos de posibles aspectos que pueden adaptarse a un abordaje tutorizado por parte de los estudiantes, dejándolos como temas abiertos o siguiendo metodologías docentes más estructuradas. En nuestra actividad académica recurrimos a noticias de prensa de forma incidental en las asignaturas de Introducción a la Medicina, Documentación y Método científico (obligatoria de primer año del grado en Medicina), Farmacología Fundamental (obligatoria de tercer año) y Farmacología Social (optativa de tercer año) $)^{9,10,23}$.

El primero de los ejemplos, referido a la muerte del dirigente serbio Milosevic, independientemente de los interrogantes detectivescos sobre la autoría del hecho en sí, es relevante el problema médico que se plantea: la muerte puede

Rev. Med. Cine. 2020; 16(3), 223-234 Ediciones Universidad de Salamanca / @®@ J. Med. Mov., 2020; 16 (3), $223-234$ 
NOTICIAS SOBRE MEDICINA Y SALUD EN UN DIARIO DE DIFUSIÓN NACIONAL.

POTENCIAL UTILIDAD EDUCATIVA EN ESTUDIANTES DE CIENCIAS DE LA SALUD MARÍA GONZÁLEZ-GARCÍA; BEGOÑA CANTABRANA; AGUSTÍN HIDALGO

ser imputada a una interacción medicamentosa con desenlace fatal porque la rifampicina, un conocido inductor enzimático, no prescrito por los médicos que tienen la responsabilidad de los cuidados del fallecido, puede aumentar el metabolismo de los antihipertensivos (no especificados) utilizados en el control de la tensión arterial ${ }^{24}$. Orientativamente puede proponerse a los estudiantes el recorrido por las tareas propuestas en la tabla 2. El desarrollo por los estudiantes requiere conocimientos de farmacología fundamental por lo que puede plantearse en tercer año del grado o en cursos superiores, dependiendo del desarrollo de los planes de estudios de cada universidad.

Otro de los ejemplos incide en la relación entre las condiciones sociales y la salud a propósito de un artículo de prensa en la que los autores plantean la relación entre la crisis económica que se consolidó a partir de 2008 (Tabla 3) y el incremento de algunas patologías y del consumo de medicamentos. Se trata de un problema recurrente que no deja de perder vigencia; antes bien se consolida ${ }^{25}$ con una mayor aparición de enfermedades que empiezan en la inestabilidad laboral y evolucionan, desde el miedo al desempleo o a no encontrar trabajo, a desarrollar ansiedad, ira y depresión. Por estas razones, la crisis económica se ha convertido en una enfermedad social y ha producido cambios en los estilos de vida generando, por ejemplo, retraimiento social, es decir, la gente no sale para no gastar ${ }^{26}$. Además, ha generado un nuevo castigo moral de la sociedad, en este caso contra los pobres, para cuyo comportamiento Adela Cortina ${ }^{27}$ ha propuesto la denominación de aporofobia.

Archibald E. Garrod, en su libro Errores innatos del metabolismo, escribe: "Cuando discutamos con más detalle los diferentes errores innatos del metabolismo conocidos, se verá que, en cada uno de ellos, la causa más probable sea la carencia congénita de alguna enzima en particular, en cuya ausencia se bloquea un proceso metabólico...». Posteriormente, Linus Pauling en 1949 descubre que la anemia de células falciformes se debe a la presencia de una globina anormal, lo que establece una relación directa causa-efecto entre la presencia de hemoglobina anormales y las consecuencias patológicas de la enfermedad de células falciformes; es decir, un cambio producido en una molécula proteica, secundario a una modificación (mutación) en el gen involucrado en la síntesis, produce una enfermedad ${ }^{28,29}$. Nace así la medicina molecular que hoy se encuentra en una fase de desarrollo acelerado y está capitalizando tanto el diagnóstico molecular como la denominada medicina de precisión, y ha otorgado un papel tal vez crucial a los laboratorios de genética aplicada, a las asociaciones de pacientes y a modalidades de financiación de la investigación desconocidas hasta hace poco tiempo.

La historia que cuentan Emilio de Benito y María Lillo ${ }^{20}$ (Tabla 4) puede servir a los estudiantes para identificar aspectos críticos del tratamiento de las enfermedades huérfanas tales como la necesidad de un mapa genético de la enfermedad, el paso de la identificación de un gen responsable al diseño de una prueba diagnóstica y plantear un tratamiento, además de las consideraciones éticas que plantean estas intervenciones (tales como la posibilidad de evitar el riesgo de concebir hermanos con la misma enfermedad, las consecuencias de diagnósticos precoces asintomáticos) o el retorno social de la inversión en investigación aplicada a un tema en el que la pasión científica y la beneficencia se dan la mano.

Por último, hemos incluido una noticia ${ }^{21}$ que demuestra que desde ópticas diferentes a la estrictamente asistencial puede detectarse el deseo de una atención centrada en el enfermo y la invocación de que el centro de toda investigación en salud debe estar orientada al beneficio del enfermo.

En síntesis, consideramos que las noticias de prensa tienen potencial interés formativo en estudiantes de ciencias de la salud por los motivos ya indicados y que se adaptan bien a la utilización de diferentes metodologías docentes. 


\section{NOTICIAS SOBRE MEDICINA Y SALUD EN UN DIARIO DE DIFUSIÓN NACIONAL. POTENCIAL UTILIDAD EDUCATIVA EN ESTUDIANTES DE CIENCIAS DE LA SALUD MARÍA GONZÁLEZ-GARCÍA; BEGOÑA CANTABRANA; AGUSTÍN HIDALGO}

\section{Referencias}

1. Revuelta G, Minelli de Oliveira J. La salud y la biomedicina en la prensa diaria. Un análisis de diez años. Periodística 2008; (11): 55-67.

2. López Cerezo JA, Gómez González J, editores. Apropiación social de la ciencia. Madrid: Biblioteca Nueva; 2008.

3. González-Alcalde G, Valderrama-Zurián JC, Aleixandre-Benavent. La investigación sobre la divulgación de la ciencia en España: situación actual y retos para el futuro. Arbor 2009; 85: 861-9.

4. López Cerezo JA. La confianza en la sociedad del riesgo. Barcelona: Sello Editorial, 2018.

5. Lobera J, editor. Percepción social de la ciencia y la tecnología 2016. Madrid: Fundación Española para la Ciencia y la Tecnología, FECYT; 2017.

6. Young E, Norman R, Humphreys R. Medicine in the popular press: the influence of the media on perceptions of disease. Plos One 3(10). e3532.

7. Aiestaran A, Camacho I, Ronco M. La información sobre salud y medicina en los diarios de la Comunidad Autónoma Vasca y Navarra. Ámbitos. 2014; (26):1-19.

8. Ronco López M, Peñafiel C, Echegaray L. El periodismo de salud en España. La información de salud en la prensa española (2000-2010): aproximación a los estudios documentales existentes. Documentación de las ciencias de la información. 2014; 37: 267-304.

9. Cantabrana B, Diez B, Bordallo J, Sanchez M, Hidalgo A. Apropiación de terminología médica por estudiantes de primer curso del grado en medicina a través de prensa diaria. FEM 2013; 16(3):145-51.

10. Cantabrana B, Diez B, Hidalgo A. Percepción por los estudiantes de la divulgación científica en la prensa y de su contribución a la cultura científica. FEM 2015; 18(1): 47-53.

11. García Sánchez JE, Trujillano Martín I, García Sánchez E. Medicina y Cine ¿Por qué? Rev Med Cine 2005; 1(1): 1-2.

12. De la Torre T. Medicina in televisión series. Fundación Dr. Antonio Esteve, 2017.

13. Hidalgo A, Bordallo J, Cantabrana B. Utilidad potencial de las artes visuales en la enseñanza de la medicina. Educ Med. 2018; 19(S3): 284-93.
14. Mayor Serrano Ma B B. El comic como recurso didáctico en los estudios de medicina. Fundación Dr. Antonio Esteve, 2016.

15. González M, Hidalgo A. Utilización de noticias de prensa en la enseñanza de la medicina. Rev Med Cine. 2020 (en prensa).

16. González-García M, Cantabrana, Hidalgo A. Ciencia, medicina y medicamentos en la prensa diaria: estudio del periodo 2001-2016. FEM. 2020; 23(1): 17-24.

17. Ferrer I. El tribunal investiga quién suministró en prisión a Milosevic fármacos no recetados. El País. 14 de marzo de 2006.

18. Mata P. Medicamento sospechoso. El País. 4 de marzo de 2006.

19. Nogueira C. Pandemia de pesimismo. El País. 11 de mayo de 2012.

20. Benito E de, Lillo M. Los enfermos «raros» toman las riendas. El País. 27 de febrero de 2014.

21. Sampedro J. No se olviden del enfermo. El País, 25 de julio de 2006.

22. Díez E, Alonso M, Bordallo J, Cantabrana B, Hidalgo A. Competencias de investigación en el Grado en Medicina en la universidad española. FEM. 2019; 22(6): 279-86.

23. González-Rodríguez $S$, Cantabrana B, Menéndez L, Hidalgo A. Aspectos sociales del medicamento en el grado en Medicina de la Universidad de Oviedo. Educ Med. 2019 (En prensa).

24. Cozza KL, Armstrong SC, Oesterheld JR. Principios de interacción farmacológica para la práctica médica. Barcelona: Ars Medica; 2006.

25. Nicieza-García ML, Alonso-Lorenzo JC, Suárez-Gil $\mathrm{P}$, Rilla-Vilar N. Efecto de la crisis económica sobre el consumo de psicofármacos en Asturias. Gac Sanit 2016; 30: 464-67.

26. Bauman Z. Daños colaterales. Madrid: Fondo de Cultura Económica; 2011.

27. Cortina A. Aporofobia, el rechazo al pobre. Un desafío para la democracia. Barcelona: Paidós; 2017.

28. García Barreno P. De pócimas y Chips. Madrid: Espasa Calpe; 2006.

29. Barona Vilar JL. Salud, tecnología y saber médico. Madrid: Centro de estudios Ramón Areces; 2004.

Rev. Med. Cine. 2020; 16(3), 223-234 Ediciones Universidad de Salamanca / @®@ J. Med. Mov., 2020; 16 (3), $223-234$ 
NOTICIAS SOBRE MEDICINA Y SALUD EN UN DIARIO DE DIFUSIÓN NACIONAL.

POTENCIAL UTILIDAD EDUCATIVA EN ESTUDIANTES DE CIENCIAS DE LA SALUD

MARÍA GONZÁLEZ-GARCÍA; BEGOÑA CANTABRANA; AGUSTÍN HIDALGO

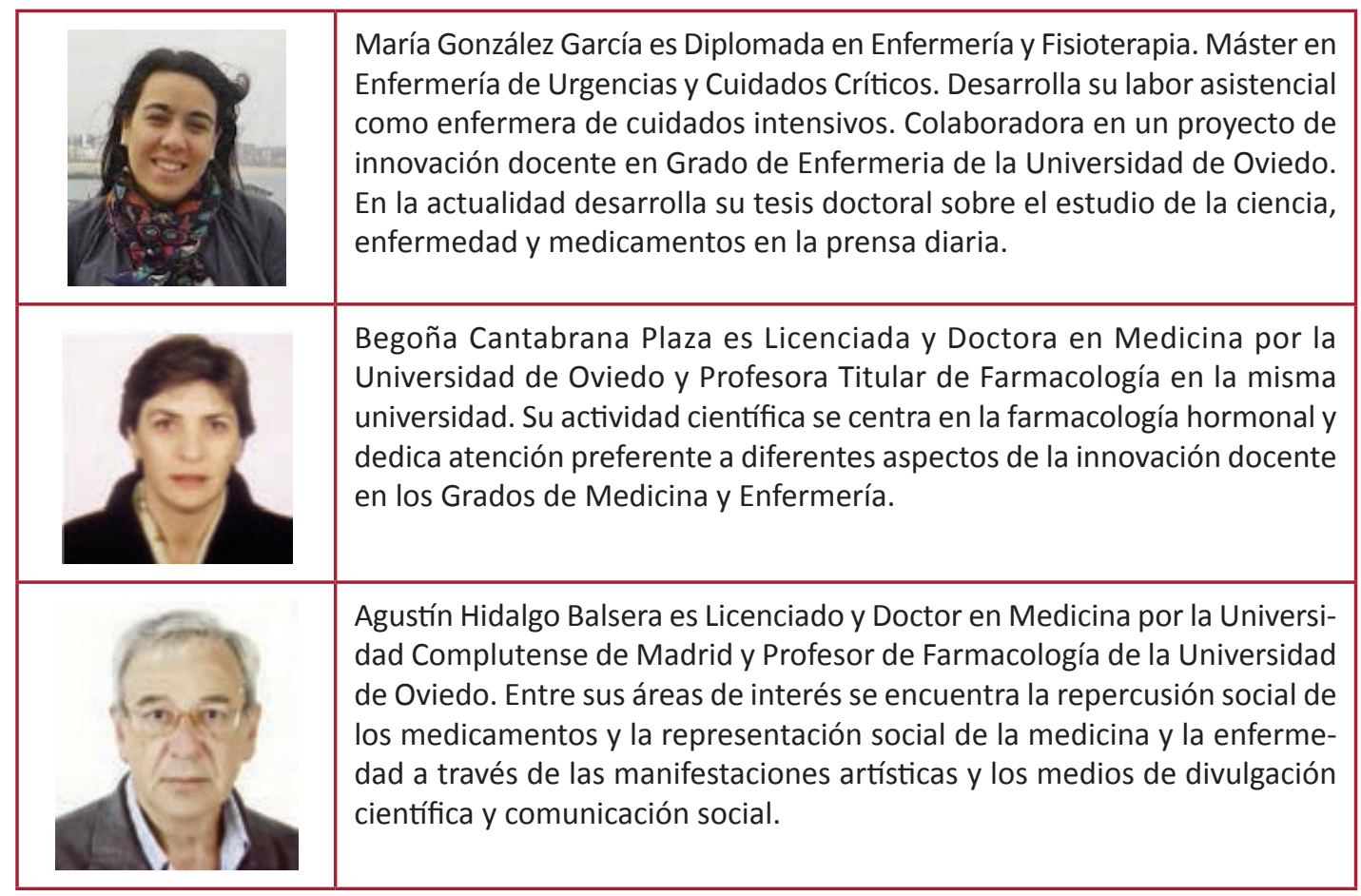

Rev. Med. Cine. 2020; 16(3), 223-234 Ediciones Universidad de Salamanca / @®@

J. Med. Mov., 2020; 16 (3), 223-234 